\title{
Frontières
}

\section{Diversité et vieillissement}

\section{Isabelle Wallach}

Volume 25, numéro 1, automne 2012

Le vieillissement et sa diversité

URI : https://id.erudit.org/iderudit/1018228ar

DOI : https://doi.org/10.7202/1018228ar

Aller au sommaire du numéro

Éditeur(s)

Université du Québec à Montréal

ISSN

1916-0976 (numérique)

Découvrir la revue

Citer ce document

Wallach, I. (2012). Diversité et vieillissement. Frontières, 25(1), 5-9.

https://doi.org/10.7202/1018228ar

Ce document est protégé par la loi sur le droit d'auteur. L'utilisation des services d'Érudit (y compris la reproduction) est assujettie à sa politique d'utilisation que vous pouvez consulter en ligne.

https://apropos.erudit.org/fr/usagers/politique-dutilisation/
Cet article est diffusé et préservé par Érudit.

Érudit est un consortium interuniversitaire sans but lucratif composé de l’Université de Montréal, l'Université Laval et l'Université du Québec à Montréal. Il a pour mission la promotion et la valorisation de la recherche. https://www.erudit.org/fr/ 


\section{DIVERSITÉ ET VIEILLISSEMENT}

Isabelle Wallach, Ph. D., Professeure au Département de sexologie, Université du Québec à Montréal

Le vieillissement de la population constitue un enjeu de plus en plus présent dans la société et les médias québécois. Souvent présenté sous un jour alarmiste, il est dépeint de façon peu nuancée en occultant la multitude de ses réalités. Pourtant, vieillir ne signifie pas faire rupture avec son passé pour entrer dans une catégorie à part de la population. Au contraire, le vieillissement s'inscrit le plus souvent dans la continuité d'une existence marquée par son individualité et par son appartenance à différents groupes. Les recherches récentes en gérontologie sociale nous mettent en garde contre la propension à réduire l'identité des aînés à la seule dimension de l'âge. De plus en plus de travaux en sciences sociales s'intéressent ainsi à la problématique de l'hétérogénéité et de la complexité du vieillissement. Ce numéro de la revue Frontières sur le vieillissement et sa diversité s'inscrit dans cette perspective et entend offrir une visibilité à des populations âgées souvent marginalisées ou oubliées. Qu'ils relèvent d'une réflexion générale sur les conditions de vie de populations âgées minoritaires ou exclues de la société, sur les aspects structurels sous-jacents à leur situation à l'étape de la vieillesse, sur leurs difficultés et besoins spécifiques ou sur leur vécu du vieillissement, les articles réunis dans ce numéro ont à cœur de comprendre et d'analyser les expériences et les enjeux spécifiques auxquels font face les personnes âgées appartenant à des groupes vulnérables ou à tout le moins non dominants.

Une des problématiques transversales à ce numéro de Frontières concerne en effet l'exclusion sociale. Si faire partie du groupe des personnes âgées expose au risque d'être progressivement mis à l'écart de la société, quel peut être le vécu d'aînés se situant à l'intersection d'autres positionnements sociaux qui leur assignent une place d'emblée peu valorisée? Afin de mieux saisir les enjeux relatifs à 
l'exclusion des aînés, ce numéro s'ouvre sur un article de Véronique Billette et de ses collègues qui proposent une analyse approfondie du concept d'exclusion sociale, envisagé dans ses multiples dimensions, mais aussi de l'inclusion sociale et de la reconnaissance des différents segments de la population âgée. Dépassant une approche purement discursive, cet article offre trois grandes avenues pour contribuer à I'inclusion des personnes âgées, traitant tour à tour du regard porté sur les personnes âgées, sur leurs milieux de vie et sur les politiques sociales et économiques qui régissent l'existence des aînés. En considérant les niveaux micro, méso et macro-social, ce cadre d'analyse accroît les possibilités de comprendre et d'agir sur les facteurs limitant la reconnaissance et l'accès aux ressources des personnes âgées. Nous invitant à considérer aussi bien les détails du quotidien et les relations interpersonnelles des aînés que les dimensions environnementales et politiques qui modulent et contraignent leur existence, cet article se révèle des plus stimulants pour susciter la réflexion et porter un regard renouvelé sur le vieillissement des individus et des groupes marginalisés.

Le rôle joué par les politiques dans les situations d'exclusion de certains segments de la population âgée est traité dans deux articles de ce numéro, l'un portant sur les itinérants et le second, sur les services en santé mentale. Burns et ses collègues mettent tout d'abord en exergue les effets des politiques publiques sur l'exclusion des personnes âgées itinérantes au Québec. Un des apports majeurs de leur article réside dans la remise en cause de la tendance prédominante à attribuer l'itinérance à des défaillances individuelles et dans son attention portée aux facteurs structurels participant à ce phénomène. Après avoir dressé un portait des multiples difficultés rencontrées par les personnes âgées itinérantes dans leur accès aux services sociaux et d'hébergement, les auteurs analysent les impacts des politiques canadiennes fédérale, provinciale et municipale (Montréal), sur la persistance du phénomène de l'itinérance des aînés. L'emphase mise par ces politiques sur l'intégration au marché du travail et l'absence de mesures ciblant spécifiquement les itinérants âgés contribuent largement à leur exclusion de la société. Ces politiques, basées sur une logique économique valorisant la productivité et I'indépendance, occultent ainsi totalement la réalité et les besoins des itinérants âgés et contribuent dès lors à les exclure de programmes pourtant supposés soutenir les plus vulnérables. 
Présenté dans la section Intervention, le texte de Kareen Nour, Véronique Billette et Alan Regenstreif, questionne pour sa part l'impact des changements de la politique en santé mentale du Québec sur les services offerts à la clientèle âgée au sein des Centres de services sociaux et de santé (CSSS). Analysant l'évolution des services spécifiques en santé mentale offerts aux personnes de 60 ans et plus par le CSSS Cavendish, suite à I'adoption du Plan en santé mentale 2005-2010 du ministère de la Santé et des Services sociaux du Québec, l'article met en lumière plusieurs changements notables pouvant être attribués à ces nouvelles orientations économiques et philosophiques. Les auteurs font ainsi le constat d'une diminution de l'approche psychosociale au profit d'une approche médicale, parallèlement à une emprise croissante des impératifs économiques de rentabilité qui contribuent à la détérioration de la qualité et de la disponibilité des services en santé mentale offerts aux aînés. Autrefois admis dans les services spécialisés en santé mentale pour la clientèle de 60 ans et plus sur la base de comportements à risques, les aînés doivent désormais avoir reçu un diagnostic psychiatrique pour en bénéficier. De plus, axé sur la réadaptation sociale, le nouveau modèle thérapeutique privilégié vise avant tout le retour à l'autonomie et au travail, ce qui paraît très éloigné des besoins des personnes âgées. Les auteurs démontrent ainsi qu'en accordant peu d'attention aux enjeux spécifiques associés au vieillissement, un tel modèle risque d'amplifier les stéréotypes âgistes sur l'inadaptation de la clientèle aînée ou l'inutilité de les traiter.

Dans la continuité de ces analyses sur la mise à l'écart institutionnelle de certains segments de la population âgée, l'article de Billy Hébert, Line Chamberland et Mickaël Chacha Enriquez, dresse un portrait de la situation encore méconnue des aînés trans en ce qui a trait à la santé et aux besoins de services. Après avoir fait état de la diversité inhérente à la population des aînés trans tant par leurs caractéristiques sociodémographiques que par leur parcours relatif à la transidentité, les auteurs s'attardent sur les nombreux problèmes de santé et les difficultés psychosociales auxquels ils sont confrontés. Bien que ces besoins de santé et de soutien psychosocial rendent essentiels leurs rapports avec les professionnels et leur accès aux services, I'article met en relief les nombreuses barrières auxquels se heurtent les aînés trans au sein du réseau de la santé et des services sociaux, ainsi que les préjugés et les attitudes discriminatoires dont font preuve les professionnels dans le réseau par ignorance ou manque de sensibilisation aux réalités de cette minorité sexuelle. 
Dans la section Recherche, la santé d'une autre minorité sexuelle âgée, celle des hommes gais et bisexuels, fait l'objet de l'article écrit par Joseph Lévy et ses collègues. Dans une première partie, une recension des écrits portant sur la santé des personnes homosexuelles âgées au Canada identifie les nombreux problèmes de santé physique et mentale qui touchent cette population, dont plusieurs apparaissent reliés à la stigmatisation de leur orientation sexuelle. L'homophobie peut également nuire à l'accès aux services de santé pour les lesbiennes et les gais âgés ou être à l'origine d'attitudes négatives de la part des professionnels. Les auteurs présentent ensuite les résultats de leur enquête pancanadienne sur la santé des minorités sexuelles, incluant plus de 400 participants hommes, homosexuels et bisexuels, de 55 ans et plus. L'article examine tant les profils de santé physique, sexuelle et mentale de cette population, leurs préoccupations relatives à la santé et aux relations interpersonnelles, tout en comparant les résultats concernant les 55-64 ans avec ceux relatifs aux 65 ans et plus. Pointant les spécificités des résultats de cette étude au regard des autres travaux sur la santé des minorités sexuelles âgées, les auteurs signalent l'importance des problématiques qui relèvent de la santé mentale des participants à cette enquête pancanadienne mais aussi les différences significatives existant entre les deux groupes d'âge.

S'éloignant des problématiques relatives à la santé et à l'accès aux services des ainés issus de populations minoritaires ou marginales, deux autres articles de la section Recherche, basés sur des recherches qualitatives, centrent leur regard sur le vécu du rapport au temps et du vieillissement des personnes âgées atteintes de pathologies graves. S'intéressant à la population des femmes âgées atteintes du cancer, Valérie Bourgeois-Guérin examine comment la souffrance se trouve profondément enchâssée dans l'interprétation du temps. À la lumière des écrits philosophiques de Ricœur, l'auteure nous propose une lecture originale du vécu de huit femmes âgées atteintes du cancer, qu'elle relie à leur perception du temps passé, présent et futur. Cette analyse centrée sur la temporalité offre une analyse novatrice des expériences difficiles vécues par les aînées et des moyens qu'elles mettent en œuvre pour composer avec cette épreuve. L'annonce d'un diagnostic de cancer constitue ainsi un événement (source de souffrance pour les aînées) qui peut les empêcher de connecter leur histoire passée à leur présent, tandis que leur perception du futur, associé à la mort, les contraint à se centrer sur le moment présent qui peut devenir une source de désespoir. 
Valérie Bourgeois-Guérin nous invite donc à repenser la souffrance des personnes âgées qui sont atteintes de maladies incurables. Elle réinscrit dans un contexte plus large leur vie passée et leur vision de l'avenir, car leur expérience présente du vieillissement est conditionnée par leur capacité à donner du sens et une continuité à ces trois temps de leur existence.

Portant sur une autre population âgée atteinte d'une pathologie grave, les personnes vivant avec le VIH, le texte d'Isabelle Wallach explore également les liens entre maladie et expérience du vieillissement mais centre son attention davantage sur le rapport au corps. Prenant en considération la dévalorisation de la vieillesse et de ses signes physiques au sein de notre société, l'auteure se questionne sur l'expérience du vieillissement de ces personnes atteintes d'une pathologie longtemps associée à la mort et qui, à I'heure actuelle, connaissent un vieillissement physique prématuré. Son analyse d'entrevues, réalisées avec 38 hommes et femmes vivant avec le VIH de 50 ans et plus, s'articule autour de trois dimensions: le vieillissement physique, le sentiment de vieillir et le rapport au vieillissement. S'intéressant aux liens entre les signes physiques de vieillissement et la reconnaissance du sentiment de vieillir, ainsi qu'aux facteurs contribuant à l'acceptation ou au refus du vieillissement, l'auteure questionne autant l'influence du genre et de l'orientation sexuelle que celle du parcours de vie avec le VIH sur l'expérience du vieillissement de cette population. Les analyses des discours des personnes âgées vivant avec le VIH nous révèlent notamment que, loin d'engendrer uniquement un vécu négatif du vieillissement, leur parcours avec la maladie peut au contraire contribuer à son acceptation.

Loin des images d'Épinal d'une vieillesse dorée et uniforme, les différents articles réunis dans ce numéro de Frontières dressent donc un portrait des réalités vécues par plusieurs populations âgées peu visibles dans les médias et dans notre société. Sans prétendre traiter de l'ensemble de la problématique du vieillissement et de la diversité, ce numéro espère avant tout susciter la réflexion, en rappelant l'importance des facteurs structurels dans les difficultés rencontrées par les aînés qui sont marginalisés et la nécessité de considérer l'intersection des multiples positionnements sociaux des personnes âgées pour mieux saisir le caractère unique et spécifique de leurs expériences du vieillissement. 